Mathematical Research Letters 9, 47-63 (2002)

\title{
K3 SURFACES VIA ALMOST-PRIMES
}

\author{
Keist Oguiso
}

\begin{abstract}
Based on the result on derived categories on K3 surfaces due to Mukai and Orlov and the result concerning almost-prime numbers due to Iwaniec, we remark the following fact: For any given positive integer $N$, there are $N$ (mutually non-isomorphic) projective complex K3 surfaces such that their Picard lattices are not isomorphic but their transcendental lattices are Hodge isometric, or equivalently, their derived categories are mutually equivalent. After reviewing finiteness result, we also give an explicit formula for the cardinality of the isomorphism classes of projective K3 surfaces having derived categories equivalent to the one of $X$ with Picard number 1 in terms of the degree of $X$.
\end{abstract}

\section{$\S 1$. Introduction}

Our main results are (1.7) - (1.10) and Proposition A in the appendix.

(1.1) Let $\mathbf{N}:=\{1,2,3, \cdots\}$ be the set of natural numbers. We call $p \in \mathbf{N}$ prime if $n \mid p$ and $n \in \mathbf{N}$ then $n=1$ or $p$. In this note it is convenient to consider the number 1 is also prime and we shall do so. There is a long standing conjecture since Dirichlet concerning primes:

Conjecture. Set $\mathcal{N}_{1}:=\left\{n \in \mathbf{N} \mid n^{2}+1\right.$ is prime $\}$. Then $\left|\mathcal{N}_{1}\right|=\infty$.

Contrary to its appearance, this conjecture is very difficult and is completely open till now. The products of two (not necessarily distinct) primes are called almost-primes. The best result known now is the following very deep Theorem due to H. Iwaniec:

Theorem (1.2) [Iw]. Let $f(x)=a x^{2}+b x+c$ be an irreducible polynomial in $\mathbf{Z}[x]$ such that $c \equiv 1(2)$ and $a>0$. Then for each such an $f$ the set of almostprimes of the form $f(n)(n \in \mathbf{N})$ are infinite. In particular, almost-primes of the form $n^{2}+1$ are infinite.

This Theorem concerning quadratically represented almost-primes is one of the most crucial ingredients of this note. In particular, we apply one special case of this Theorem, namely, the case $f(x)=4 x^{2}+1$ for our study of K3 surfaces. (The argument also goes through if we take $f(x)=x^{2}+1$, but then we sometimes need case by case description according to the congruence mod 4 .

Received October 31, 2001.

2000 Mathematics Subject Classification 14J28. 
This is only the reason why we choose $4 x^{2}+1$ instead of more attractive $x^{2}+1$.) We set

$$
\mathcal{N}_{2}:=\left\{n \in \mathbf{N} \mid 4 n^{2}+1 \text { is almost prime }\right\} .
$$

By $(1.2),\left|\mathcal{N}_{2}\right|=\infty$. If $n \in \mathcal{N}_{2}$, then we have the factorization $4 n^{2}+1=p q$. Note that $4 n^{2}+1 \equiv 1 \bmod 4, p \equiv q \equiv 1 \bmod 2$ and $p \neq q$; Indeed, if $p=q$, then $1=(p-2 n)(p+2 n)$, a contradiction to $p+2 n \geq 2$.

(1.3) Throughout this note, a K3 surface means a 2-dimensional complex projective smooth variety which admits nowhere vanishing global 2-form but admits no nonzero global one form. Let $X$ be a $\mathrm{K} 3$ surface. Let $(*, * *)$ be the symmetric bilinear form on $H^{2}(X, \mathbf{Z})$ given by the cup product. Then $\left(H^{2}(X, \mathbf{Z}),(*, * *)\right)$ is an even unimodular lattice of signature $(3,19)$. We denote by $\mathrm{NS}(X) \simeq$ Pic $X$ the Néron-Severi lattice of $X$ and by $\rho(X)$ the Picard number, i.e. rank of $\mathrm{NS}(X)$. This lattice $\mathrm{NS}(X)$ is primitive in $H^{2}(X, \mathbf{Z})$ and is of signature $(1, \rho(S)-1)$. We call the orthogonal lattice of $\mathrm{NS}(X)$ in $H^{2}(X, \mathbf{Z})$ the transcendental lattice and denote it by $T_{X}$, i.e.

$$
T_{X}:=\left\{x \in H^{2}(X, \mathbf{Z}) \mid(x, y)=0 \text { for all } y \in \mathrm{NS}(X)\right\} .
$$

$T_{X}$ is also primitive in $H^{2}(X, \mathbf{Z})$ and is of signature $(2,20-\rho(X))$. Let $\omega_{X}$ be a non-zero global 2-form on $X$. Then, one has the inclusion

$$
\mathbf{C} \omega_{X} \oplus \mathbf{C} \bar{\omega}_{X} \subset T_{X} \otimes_{\mathbf{Z}} \mathbf{C} .
$$

This inclusion defines the Hodge structure of weight 2 on $T_{X}$. This is a subHodge structure of the Hodge structure of $H^{2}(X, \mathbf{Z})$. We refer to the reader $[\mathrm{BPV}]$ for more details. Based on the pioneering work of Mukai [Mu], Orlov [Or] proved the following beautiful Theorem:

Theorem (1.4) [Or Theorem (3.3)]. Let $X_{1}, X_{2}$ be K3 surfaces. Then the following three statements are equivalent to one another:

(1) There is a Hodge isometry between $T_{X_{1}}$ and $T_{X_{2}}: \varphi_{12}:\left(T_{X_{1}}, \mathbf{C} \omega_{X_{1}}\right) \rightarrow$ $\left(T_{X_{2}}, \mathbf{C} \omega_{X_{2}}\right)$.

(2) The bounded derived categories of choherent sheaves $D\left(X_{1}\right)$ and $D\left(X_{2}\right)$ are equivalent as triangulated category.

(3) $X_{2}$ is isomorphic to (the base space of) a 2-dimensional compact fine moduli space of some stable sheaves on $X_{1}$.

Concerning the derived categories, we refer the reader to an excellent book $[\mathrm{GM}]$. Although the equivalence (1) and (3) is implicit in [Or], his proof goes as $(2) \rightarrow(1) \rightarrow(3) \rightarrow(2)$. See also [BM 1, 2], [Cl], [Th] for further progress and relevant work.

(1.5) In their paper [BO], Bondal and Orlov observed that an equivalence class of the derived category $D(V)$ recovers the original manifold $V$ if $V$ is a Fano manifold or a manifold of ample canonical sheaf. Theorem (1.4) says that one can at least recover the transcendental lattice and the period inside the transcendental lattice if a manifold is a K3 surface. Therefore it is natural to ask: 


\section{Question (1.6).}

(1) to what extent does the equivalence class of $D(X)$, or equivalently by (1.4), the Hodge isometry class of $T_{X}$, recover the original K3 surface $X$ ?

(2) at least to what extent does the equivalence class of $D(X)$ recover the algebraic part of the lattice $N S(X)$ ?

In this direction, Mukai [Mu, Proposition (6.2)] already observed that if $\rho(X) \geq 12$, i.e. if $\operatorname{rank} T_{X} \leq 10$, then the equivalence of $D(X)$ recovers $X$ itself.

The aim of this note is to prove the following result:

Main Theorem (1.7). Let $N$ be an arbitrarily given natural number. Set $I_{N}:=\{1,2, \cdots, N\}$ and $\Delta_{N}:=\{(i, i) \mid i \in I\}$. Then there are $N$ K3 surfaces $X_{i}$ $\left(i \in I_{N}\right)$ depending on $N$ such that

(1) for any $(i, j) \in I_{N}^{2}-\Delta_{N}$, the Néron-Severi lattices $N S\left(X_{i}\right)$ and $N S\left(X_{j}\right)$ are not isomorphic, therefore $X_{i} \neq X_{j}$ as well, but such that

(2) there is a Hodge isometry $\varphi_{i j}:\left(T_{X_{i}}, \mathbf{C} \omega_{X_{i}}\right) \rightarrow\left(T_{X_{j}}, \mathbf{C} \omega_{X_{j}}\right)$ for any $(i, j) \in I_{N}^{2}$.

We shall construct such $X_{i}$ of $\rho\left(X_{i}\right)=2$. Note that 2 is the least possible Picard number for such examples to exist. Indeed, if $\rho(X)=1$, then NS $(X)=\mathbf{Z} l$ and $\left(l^{2}\right)=\left|\operatorname{det} T_{X}\right|$ and therefore NS $(X)$ are isomorphic if so are $T_{X}$. See (1.10) for the extent to which the Hodge structure of $T_{X}$ recovers $X$ when $\rho(X)=1$. This is related to the factorization of $\operatorname{deg} X$. See also Proposition $\mathrm{A}$ in the appendix in higher dimensional case.

Using the global Torelli Theorem of K3 surfaces and Gauss' Theorem of quadratic forms, we can reduce the Theorem to a problem of algebraic number theory: find a sequence of real quadratic fields $\mathbf{Q}(\sqrt{m})$ having large class number $h(m)$ compared with a cardinality $q(m)$ of possible discriminant forms, i.e. $\mathbf{Q} / 2 \mathbf{Z}$-valued quadratic forms on the discriminant groups. However, there is no relation between the growth of $h(m), q(m)$ and $m$ even if $m$ is square free. Indeed, there is an open problem whether $h(m)=1$ for infinitely many $\mathbf{Q}(\sqrt{m})$ since Gauss. The growth of $h(m)$ is related to the growth of the norms of the fundamental units and the growth of $q(m)$ is related to the factorization of $m$. Theorem (1.2) or the infinite set $\mathcal{N}_{2}$ gives us a way to control the quantities $h(m)$ and $q(m)$ simultaneously when $m=4 n^{2}+1$ and $n \in \mathcal{N}_{2}$. We shall prove (1.7) in Section 2.

As a direct consequence of (1.4) and (1.7), one obtains:

Corollary (1.8). For any natural number $N$,

(1) there exist at least $N$ K3 surfaces whose derived categories are equivalent but whose Picard groups are non-isomorphic.

(2) there exists a K3 surface $X$ having at least $N$ mutually non-isomorphic 2-dimensional compact fine moduli spaces of stable sheaves on $X$. 
In the opposite direction, we recall the following fact found by Bridgeland and Maciocia (with a bit more constructive proof for the use of the next Proposition (1.10)):

Proposition (1.9) [BM1, Proposition (5.3)]. Let $X$ be a K3 surface. Let $\mathcal{S}_{T}(X)$ be the set of isomorphism classes of K3 surfaces $Y$ such that there is a Hodge isometry $\left(T_{Y}, \mathbf{C} \omega_{Y}\right) \simeq\left(T_{X}, \mathbf{C} \omega_{X}\right)$. Then, $\left|\mathcal{S}_{T}(X)\right|<\infty$.

(1.9) with (1.4) says that the equivalence class of $D(X)$ recovers K3 surface $X$ up to finitely many ambiguities as manifold.

It is then interesting to seek explicit values of $\left|\mathcal{S}_{T}(X)\right|$. We shall carry out this in the most generic case, i.e. for K3 surfaces with Picard number 1. Here we write the result in terms of moduli via (1.4):

Proposition (1.10). Let $X$ be a K3 surface with $N S(X)=\mathbf{Z} l_{X}$. Set $\operatorname{deg} X=$ $\left(l_{X}^{2}\right)=2 n$. Let $m$ be the number of non-isomorphic 2-dimemsional compact fine moduli spaces of stable sheaves on $X$. Then $m=\left|\left((\mathbf{Z} / 4 n)^{\times}\right)_{2}\right| / 4$ if $n \neq 1$ and $m=\left|(\mathbf{Z} / 4)^{\times}\right| / 2=1$ if $n=1$. Here $(\mathbf{Z} / 4 n)^{\times}$is the unit group of the ring $\mathbf{Z} / 4 n$ and $\left((\mathbf{Z} / 4 n)^{\times}\right)_{2}$ is the two torsion subgroup of $(\mathbf{Z} / 4 n)^{\times}$. More explicitly:

(1) $m=1$ if $\operatorname{deg} X=2$;

(2) $m=1$ if $\operatorname{deg} X=2^{a}$;

(3) $m=2^{k-1}$ if $\operatorname{deg} X=2 p_{1}^{e_{1}} \cdots p_{k}^{e_{k}}$;

(4) $m=2^{k}$ if $\operatorname{deg} X=2^{a} p_{1}^{e_{1}} \cdots p_{k}^{e_{k}}$.

Here $a$ is a natural number such that $a \geq 2, p_{i}$ are mutually different primes such that $p_{i} \geq 3$, and $k$ and $e_{i}$ are natural numbers.

We note that for any given natural number $n$, there is a K3 surface $X$ such that $\rho(X)=1$ and $\operatorname{deg} X=2 n$.

\section{$\S$ 2. Proof of (1.7)}

(2.1) By a lattice $L:=(L,(*, * *))$, we mean a free abelian group $L$ of finite rank, which we write by $\mathrm{rk} L$, equipped with an integral valued non-degenerate symmetric bilinear form $(*, * *): L \times L \rightarrow \mathbf{Z}$. We write $\left(x^{2}\right)=(x, x)$. Two lattices $L_{1}=\left(L_{1},(*, * *)_{L_{1}}\right)$ and $L_{2}=\left(L_{2},(*, * *)_{L_{2}}\right)$ are said to be isomorphic or isometric if there is an isomorphism of abelian groups $f: L_{1} \rightarrow L_{2}$ such that $(f(x), f(y))_{L_{2}}=(x, y)_{L_{1}}$. A lattice $L$ is said to be even if $\left(x^{2}\right) \equiv 0 \bmod 2$. We often represent a lattice $L$ by a symmetric integral matrix $S_{L}=\left(\left(e_{i}, e_{j}\right)\right)$ and call $S_{L}$ an associated matrix to $L$. Here $\left\langle e_{i}\right\rangle_{i=1}^{\mathrm{rk} L}$ is integral basis of $L$. An associated matrix is uniquely determined by $L$ up to the action by $\mathrm{GL}(\operatorname{rk} L, \mathbf{Z})$ : $S \mapsto{ }^{t} M S M$ on the space of symmetric matrices of degree rk $L$. Note also that two lattices are isomorphic if and only if their associated symmetric matrices lie in the same orbit under the action by $\operatorname{GL}(\operatorname{rk} L, \mathbf{Z})$. We $\operatorname{define} \operatorname{det} L:=\operatorname{det} S_{L}$. This is well defined, because $\operatorname{det} M= \pm 1$. The signature $(p, q)$ of $L$ is defined to be the signature of an associated matrix $S_{L}$. Then $q=\operatorname{rk} L-p$. We call $L$ hyperbolic if the signature is $(1, \mathrm{rk} L-1)$. 
(2.2) We set $L^{*}=\operatorname{Hom}_{\mathbf{Z}}(L, \mathbf{Z})$. Using non-degenerate $(*, * *)$, one has a natural inclusions $L \subset L^{*} \subset L \otimes_{\mathbf{Z}} \mathbf{Q}$. Then, $(*, * *)$ is extended to a rational valued symmetric bilinear forms on $L \otimes \mathbf{z} \mathbf{Q}$ and on $L^{*}$. Set $A_{L}:=L^{*} / L$. We call $A_{L}$ the discriminant group of $L$. The discriminant group $A_{L}$ is a finite abelian group of order $|\operatorname{det} L|$. The bilinear form $(*, * *)$ of $L$ induces the symmetric bilinear form $B_{A_{L}}(*, * *): A_{L} \times A_{L} \rightarrow \mathbf{Q} / \mathbf{Z}$. This is defined by $B_{A_{L}}(\bar{x}, \bar{y})=$ $(x, y) \bmod \mathbf{Z}$, where $x, y \in L^{*}, \bar{x}=x \bmod L, \bar{y}=y \bmod L$. If $L$ is even, then we have a quadratic form $Q_{A_{L}}: A_{L} \rightarrow \mathbf{Q} / 2 \mathbf{Z}$. This is defined by $Q_{L}(\bar{x})=$ $\left(x^{2}\right) \bmod 2 \mathbf{Z}$. This quadratic form $Q_{A_{L}}$ satisfies

$$
\begin{gathered}
Q_{A_{L}}(n \bar{x})=n^{2} Q_{A_{L}}(\bar{x}) \\
Q_{A_{L}}(\bar{x}+\bar{y})=Q_{A_{L}}(\bar{x})+Q_{A_{L}}(\bar{y})+2 B_{A_{L}}(\bar{x}, \bar{y}) .
\end{gathered}
$$

We call $\left(A_{L}, Q_{A_{L}}\right)$ a discriminant form of $L$.

(2.3) Let $A$ be a finite abelian group. As in [Ni], $Q_{A}: A \rightarrow \mathbf{Q} / 2 \mathbf{Z}$ is called a quadratic form on $A$ if $Q_{A}$ satisfies $Q_{A}(n a)=n^{2} Q_{A}(a)$ for $a \in A$ and $n \in \mathbf{Z}$ and if there is a symmetric bilinear map $B(*, * *): A \times A \rightarrow \mathbf{Q} / \mathbf{Z}$ such that $Q_{A}(a+b)=Q_{A}(a)+Q_{A}(b)+2 B_{L}(a, b)$. Two elements $a, b \in A$ are said to be orthogonal if $B_{A}(a, b)=0$. Two pairs $\left(A, Q_{A}\right)$ and $\left(A^{\prime}, Q_{A^{\prime}}\right)$ of finite abelian groups and their quadratic forms are said to be isomorphic if there is a group isomorphism $\varphi: A \rightarrow A^{\prime}$ such that $Q_{A}(a)=Q_{A^{\prime}}(\varphi(a))$ for all $a \in A$. We define the orthogonal sum $\left(A_{1} \oplus A_{2}, Q_{A_{1}} \oplus Q_{A_{2}}\right)$ of $\left(A_{1}, Q_{A_{1}}\right)$ and $\left(A_{2}, Q_{A_{2}}\right)$ in a natural manner: That is, $A_{1} \oplus A_{2}$ is the direct sum as abelian group and $Q_{A_{1}} \oplus Q_{A_{2}}\left(a_{1} \oplus a_{2}\right):=Q_{A_{1}}\left(a_{1}\right)+Q_{A_{2}}\left(a_{2}\right)$.

Let $L$ be an even lattice. One can decompose $A_{L}$ into the direct sum of the Sylow subgroups of $A_{L}$ :

$$
A_{L}=\oplus_{i=1}^{k}\left(A_{L}\right)_{p_{i}} .
$$

Here $\left(A_{L}\right)_{p_{i}}$ is the Sylow $p_{i}$-subgroup of $A_{L}$, i.e. the maximal $p_{i}$-primary subgroup of $A_{L}$.

Lemma (2.4) [Ni, Page 108-109].

(1) If $i \neq j$, then $B_{L}\left(\bar{x}_{i}, \bar{x}_{j}\right)=0$ for $\bar{x}_{i} \in\left(A_{L}\right)_{p_{i}}$ and $\bar{x}_{j} \in\left(A_{L}\right)_{p_{j}}$.

(2) Let $x \in L^{*}$ and write $\bar{x}=\sum_{i=1}^{k} \bar{x}_{i}$, where $\bar{x}_{i} \in\left(A_{L}\right)_{p_{i}}$. Then, $Q_{L}(\bar{x})=$ $\sum_{i=1}^{k} Q_{L}\left(\bar{x}_{i}\right)$. In other words, we have the orthogonal decomposition

$$
\left(A_{L}, Q_{L}\right)=\oplus_{i=1}^{k}\left(\left(A_{L}\right)_{p_{i}}, Q_{L} \mid\left(A_{L}\right)_{p_{i}}\right) .
$$

Proof. It is clear that (1) implies (2). Let us show (1). Take $\bar{x}_{i} \in\left(A_{L}\right)_{p_{i}}$ and $\bar{x}_{j} \in\left(A_{L}\right)_{p_{j}}$ and set ord $\left(\bar{x}_{i}\right)=p_{i}^{n}$. Since $p_{i}^{n}$ and $p_{j}$ are coprime, there is $\bar{y}_{j} \in\left(A_{L}\right)_{p_{j}}$ such that $\bar{x}_{j}=p_{i}^{n} \bar{y}_{j}$. Then one calculates

$$
B_{L}\left(\bar{x}_{i}, \bar{x}_{j}\right)=B_{L}\left(\bar{x}_{i}, p_{i}^{n} \bar{y}_{j}\right)=B_{L}\left(p_{i}^{n} \bar{x}_{i}, \bar{y}_{j}\right)=B_{L}\left(0, \bar{y}_{j}\right)=0 .
$$


Lemma (2.5). Let $p \neq q$ be odd primes. Set $\mathcal{L}_{p, q}$ to be the isomorphism classes of even hyperbolic lattices $L$ such that $|\operatorname{det} L|=p q$. Set $\mathcal{Q}_{p, q}$ to be the set of the isomorphism classes of $\left(A_{L}, Q_{A_{L}}\right)$ such that $L \in \mathcal{L}_{p, q}$. Then there is a positive integer $B$ being independent of $p, q$ such that $\left|\mathcal{Q}_{p, q}\right| \leq B$ for all $p \neq q$.

Proof. Let $L \in \mathcal{L}_{p, q}$. Then the decomposition of $A_{L}$ into the Sylow subgroups is $\mathbf{Z} / p \oplus \mathbf{Z} / q$. By (2.4), one has $Q_{L}=Q_{p} \oplus Q_{q}$, where $Q_{p}$ and $Q_{q}$ are quadratic forms on $\mathbf{Z} / p$ and $\mathbf{Z} / q$ respectively. It suffices to estimate the cardinality of the isomorphism classes $\left(\mathbf{Z} / p, Q_{p}\right)$. Note that $Q_{p}$ is determined by the value $Q_{p}(1)$.

If $p=1$, then there is just one quadratic form on $\mathbf{Z} / 1=\{0\}$. So, we may assume $p \geq 3$. Choose and fix an element $p_{-} \in \mathbf{N}$ such that $\left(\frac{p_{-}}{p}\right)=-1$. Here $\left(\frac{*}{p}\right)$ is the Legendre symbol of the quadratic residue, i.e. $\left(\frac{a}{p}\right)=-1$ if and only if $a$ is not a square in the multiplicative group $(\mathbf{Z} / p)^{\times}$. By $Q_{p}(p)=Q_{p}(0)=0$, one has $Q_{p}(1)=2 a / p$, where $a$ is some integer. Note that the maps $n: \mathbf{Z} / p \rightarrow \mathbf{Z} / p$ defined by $1 \mapsto n \cdot 1$ are all isomorphism as abelian group provided that $n$ is coprime to $p$ and satisfy $Q_{p}(n)=n^{2} Q_{p}(1)$. Thus, $\left(\mathbf{Z} / p, Q_{p}\right)$ is isomorphic to either $\left(\mathbf{Z} / p, q_{0}\right),\left(\mathbf{Z} / p, q_{+}\right)$or $\left(\mathbf{Z} / p, q_{-}\right)$, where $q_{*}$ are defined by $q_{0}(1)=0$, $q_{+}(1)=2 / p, q_{-}(1)=2 p_{-} / p$. Therefore one can take $B=3 \cdot 3=9$.

Let $m>1$ be a square free natural number such that $m \equiv 1(4)$. Then $\mathbf{Q}(\sqrt{m})$ is a real quadratic field. We write $\mathbf{Q}(\sqrt{m})$ by $K(m)$. Denote by $O(m), h(m)$, $\epsilon(m)>1, D(m)$ the ring of integers, the class number, the fundamental unit, the discriminant of $K(m)$ respectively. $O(m)$ is by definition, the normalization of $\mathbf{Z}$ in $\mathbf{K}(m)$ and $O(m)=\mathbf{Z}[(1+\sqrt{m}) / 2]$. One has $D(m)=m$. The unit group $O(m)^{\times}$of $O(m)$ satisfies $O(m)^{\times}=\langle\epsilon(m)\rangle \times\langle-1\rangle \simeq \mathbf{Z} \times \mathbf{Z} / 2$. Among the four possible free generators $\pm \epsilon(m)^{ \pm 1}$ of $O(m)^{\times}$, the fundamental unit is the one which is greater than 1 . The class number $h(m)$ is defined to be the cardinality of the ideal class group $C(m)$ of $O(m)$. We need the following two Theorems (2.7) and (2.8). The first one is attributed to Gauss and the second one is (a special case of) the Theorem of Siegel-Brauer. For the statement, we recall that an integral binary quadratic form $f(x, y)=a x^{2}+b x y+c y^{2}$ is called primitive if $(a, b, c)=1$. The integer $d(f):=b^{2}-4 a c$ is called the discriminant of $f$. By $S_{f}$, we denote the associated symmetric (rational) matrix, i.e.

$$
S_{f}=\left(\begin{array}{cc}
a & \frac{b}{2} \\
\frac{b}{2} & c
\end{array}\right) .
$$

Note that $\operatorname{det} S_{f}=-d(f) / 4$. We call two integral binary quadratic forms $f$ and $f^{\prime}$ are properly equivalent if $S_{f}$ and $S_{f^{\prime}}$ lie in the same orbit under the natural action by $\mathrm{SL}(2, \mathbf{Z})$, i.e. if there is $M \in \mathrm{SL}(2, \mathbf{Z})$ such that ${ }^{t} M S_{f} M=S_{f^{\prime}}$.

Theorem (2.7) (e.g. [Nr, Theorem (8.6)]). There is a natural one-to-one correspondence between the class group $C(m)$ and the set of the properly equivalent classes of primitive integral binary forms of discriminant $m$. In particular, $h(m)$ is the cardinality of the set of the properly equivalent classes of primitive integral binary forms of discriminant $m$. 


\section{Theorem (2.8) (e.g. [Nr, Theorem (8.5)]).}

$$
\lim _{m \rightarrow \infty} \frac{\log (h(m) \cdot \log \epsilon(m))}{\log D(m)}=\frac{1}{2} .
$$

The following Proposition is crucial for the main Theorem:

Proposition (2.9). Let $N$ be an arbitrarily given natural number. Set $I_{N}:=$ $\{1,2, \cdots, N\}$ and $\Delta_{N}:=\left\{(i, i) \mid i \in I_{N}\right\} \subset I_{N}^{2}$. Then, there exist $N$ even hyperbolic lattices of rank 2 , say, $S_{i}\left(i \in I_{N}\right)$ such that

(1) if $(i, j) \in I_{N}^{2}-\Delta_{N}$, then $S_{i}$ and $S_{j}$ are not isomorphic, but

(2) for all $(i, j) \in I_{N}^{2}$, the discriminant forms $\left(A_{S_{i}}, Q_{A_{S_{i}}}\right)$ and $\left(A_{S_{j}}, Q_{A_{S_{j}}}\right)$ are isomorphic.

Proof. Let $\mathcal{N}_{2}$ be the set in the Introduction. Let $n \in \mathcal{N}_{2}$ and write $d(n):=$ $4 n^{2}+1=p q$. Note that $d(n) \equiv 1 \bmod 4$. Set $K_{n}:=K(d(n)), O_{n}:=O(d(n))$, $O_{n}^{\times}=O(d(n))^{\times}, \epsilon_{n}:=\epsilon(d(n)), h_{n}:=h(d(n)), D_{n}:=D(d(n))=4 n^{2}+1=p q$ under the notation in (2.6). Recall that $\mathcal{N}_{2}$ is an infinite set.

Lemma (2.10). For any given $\epsilon>0$, there is a natural number $M$ such that

$$
\frac{\log \left(\log \epsilon_{n}\right)}{\log D_{n}}<\epsilon
$$

for all natural numbers $n$ such that $n>M$ and $n \in \mathcal{N}_{2}$.

Proof of (2.10). Consider $2 n+\sqrt{p q} \in O_{n}$. We may assume that $n \geq 3$. We have $\log \epsilon_{n}>0$ by the definition. Note that

$$
(2 n+\sqrt{p q})(2 n-\sqrt{p q})=4 n^{2}-p q=4 n^{2}-\left(4 n^{2}+1\right)=-1 .
$$

From this identity, we obtain

$$
\frac{1}{2 n+\sqrt{p q}}=-(2 n-\sqrt{p q}) \in O_{n} .
$$

In particular, $2 n+\sqrt{p q} \in O_{n}^{\times}$. Combining this with $2 n+\sqrt{p q}>1$, we find a natural number $l$ such that $(2 n+\sqrt{p q})^{2}=\epsilon_{n}^{l}$. Using $4 n \geq \sqrt{4 n^{2}+1}=\sqrt{p q}$, $n \geq 3$ and $\epsilon_{n}>1$, we calculate that

$$
\begin{gathered}
4 \log 2 n \geq 2 \log 2 n+2 \log 3=2 \log 6 n \geq 2 \log (2 n+\sqrt{p q}) \\
=\log (2 n+\sqrt{p q})^{2}=\log \epsilon_{n}^{l}=l \log \epsilon_{n} \geq \log \epsilon_{n} .
\end{gathered}
$$

We have also

$$
D_{n}=4 n^{2}+1 \geq 2 n .
$$

Combining these two inequalities, one has

$$
\frac{\log \left(\log \epsilon_{n}\right)}{\log D_{n}} \leq \frac{\log (4 \log 2 n)}{\log 2 n}=\frac{\log (\log 2 n)+\log 4}{\log 2 n} .
$$

Since $\lim _{x \rightarrow \infty} \log x=\infty$ and $\lim _{x \rightarrow \infty}(\log x) / x=0$, this gives the result.

Combining (2.8) with (2.10) applied for $\epsilon=1 / 8$, we obtain 
Corollary (2.11). There is a natural number $M$ such that

$$
\frac{\log h_{n}}{\log D_{n}}>\frac{1}{4}
$$

for all natural numbers $n$ such that $n>M$ and $n \in \mathcal{N}_{2}$. In particular,

$$
\lim _{n \rightarrow \infty, n \in \mathcal{N}_{2}} h_{n}=\infty \text {. }
$$

Let us return back to the proof of (2.9). Let $B$ be the constant in (2.5). Take an arbitrary natural number $N$. By (2.11), there is $n \in \mathcal{N}_{2}$ such that $h_{n}>2 B N$. We write $4 n^{2}+1=p q$. Then by (2.7), there are more than $2 B N$ binary forms of discriminant $p q$ which are not properly isomorphic to one another. We write them by $a_{i} x^{2}+b_{i} x y+c_{i} y^{2}, i \in\{1,2, \cdots, 2 B N\}$. These binary forms satisfy $b_{i}^{2}-4 a_{i} c_{i}=p q$. Then, the associated symmetric matrices multiplied by 2 , i.e.

$$
M_{1}=\left(\begin{array}{cc}
2 a_{1} & b_{1} \\
b_{1} & 2 c_{1}
\end{array}\right), \cdots, M_{2 B N}=\left(\begin{array}{cc}
2 a_{2 B N} & b_{2 B N} \\
b_{2 B N} & 2 c_{2 B N}
\end{array}\right)
$$

are all even, hyperbolic, and of determinant $-p q$ and lie in mutually different orbits under the natural action by $\operatorname{SL}(2, \mathbf{Z})$. Since $\operatorname{SL}(2, \mathbf{Z})$ is a normal subgroup of $\mathrm{GL}(2, \mathbf{Z})$ of index 2 , among these $2 B N$ matrices $M_{i}$, one can find $B N$ matrices lying in mutually different orbits under the action by $\mathrm{GL}(2, \mathbf{Z})$. By renumbering, we may assume they are $M_{i}(i \in\{1, \cdots, B N\})$. Then, these $B N$ matrices $M_{i}$ $(i \in\{1, \cdots, B N\})$ define $B N$ mutually non-isomorphic even hyperbolic lattices $S_{i}$ of determinant $-p q<0$ and of rank 2 . On the other hand, by (2.5), there are at most $B$ discriminant forms $\left(A_{S_{i}}, Q_{A_{S_{i}}}\right)$ up to isomorphism when $i$ runs through $\{1, \cdots, B N\}$. Therefore, among these $S_{i}(i \in\{1, \cdots, B N\})$, there are at least $B N / B=N$ lattices, say, $S_{i}(i \in\{1, \cdots, N\})$, which have mutually isomorphic discriminant forms. Now we are done for (2.9).

We shall construct K3 surfaces in the main Theorem (1.7) by using the lattices $S_{i}$ in (2.9). For this, we need the following Theorem due to Nikulin. For the statement, we recall that an embedding as lattice $\Phi: M \rightarrow L$ is said to be primitive if the abelian group $L / \Phi(M)$ is free, in other words, free basis of $\Phi(M)$ can be extended to free basis of $L$, or passing to the dual, the natural homomorphism $L^{*} \rightarrow \Phi(M)^{*}$ is surjective. A sublattice $M$ of $L$ is called primitive if the inclusion map is primitive.

Theorem (2.11) [Ni, Theorem (1.14.4), Corollary (1.13.3)]. Let $M$ be an even lattice of signature $\left(m_{+}, m_{-}\right)$(therefore $\left.r k M=m_{+}+m_{-}\right)$. Let $L$ be an even unimodular lattice of signature $\left(l_{+}, l_{-}\right)$.

(1) Let $\Phi: M \rightarrow L$ be a primitive embedding and $K$ the orthogonal lattice of $\Phi(M)$ in $L$, i.e. $K=\{x \in L \mid(x, y)=0$ for all $y \in \Phi(M)\}$. (Note that $K$ is primitive in $L$.) Then, under isomorphism

$$
M^{*} / M \simeq \Phi(M)^{*} / \Phi(M) \simeq L /(\Phi(M) \oplus K) \simeq K^{*} / K
$$


given by the natural surjective homomorphism $L^{*}=L \rightarrow \Phi(M)^{*}$ and $L^{*}=L \rightarrow K^{*}$, one has

$$
\left(A_{M}, Q_{A_{M}}\right) \simeq\left(A_{K},-Q_{A_{K}}\right)
$$

(2) Assume that $l_{+}-m_{+}>0, l_{-}-m_{-}>0$ and $r k L-r k M \geq 2+l\left(A_{M}\right)$, where $l\left(A_{M}\right)$ is the minimal number of generators of the finite abelian group $A_{M}=M^{*} / M$. Then, $M$ can be primitively embedded into $L$. Moreover, a primitive embedding $M \rightarrow L$ is unique in the sense that if $f_{i}: M \rightarrow L(i=1,2)$ are two primitive embeddings, then there is an isometry $\varphi: L \rightarrow L$ such that $f_{2}=\varphi \circ f_{1}$.

(3) Let $\tilde{M}$ be an even lattice. Assume that the signature of $\tilde{M}$ is $\left(m_{+}, m_{-}\right)$, i.e. the same as the signature of $M$ and that $\left(A_{\tilde{M}}, Q_{A_{\tilde{M}}}\right)$ is isomorphic to $\left(A_{M}, Q_{A_{M}}\right)$. Then $\tilde{M}$ is isomorphic to $M$ provided that $r k M \geq 2+$ $l\left(A_{M}\right), m_{+}>0$ and $m_{-}>0$.

Set $\Lambda:=U^{\oplus 3} \oplus E_{8}(-1)^{\oplus 2}$. Here $U$ is the lattice given by $\left(\begin{array}{cc}0 & 1 \\ 1 & 0\end{array}\right)$ and $E_{8}(-1)$ is the negative lattice given by the Dynkin diagram of type $E_{8}$. This $\Lambda$ is an even unimodular lattice of signature $(3,19)$ and is called the $K 3$ lattice. This is the unique even unimodular lattice of signature $(3,19)$ up to isomorphism. For any K3 surface $X$, there is an isometry $\tau: H^{2}(X, \mathbf{Z}) \rightarrow \Lambda$ (See for instance [BPV]). We call an isometry $\tau$ from $H^{2}(X, \mathbf{Z})$ to $\Lambda$ a marking of $X$. We call such a pair $(X, \tau)$ a marked K3 surface.

Lemma (2.12). Let $S_{i}(1 \leq i \leq N)$ be the lattices found in (2.9). Then

(1) $S_{i}$ are primitively embedded into $\Lambda$, say, $\Phi_{i}: S_{i} \rightarrow \Lambda$.

(2) Denote the orthogonal lattices of $\Phi_{i}\left(S_{i}\right)$ in $\Lambda$ by $T_{i}$. Then $T_{i}$ are isomorphic to one another.

Proof. By the elementary divisor theory, one has $l\left(A_{S}\right) \leq \operatorname{rk} S$. Since $\operatorname{rk} S_{i}=2$, $(2.11)(2)$ implies (1). Let us check (2). Since $\left(A_{S_{i}}, Q_{A_{S_{i}}}\right) \simeq\left(A_{S_{j}}, Q_{A_{S_{j}}}\right)$ by the construction, we have $\left(A_{T_{i}}, Q_{A_{T_{i}}}\right) \simeq\left(A_{T_{j}}, Q_{A_{T_{j}}}\right)$ by $(2.11)(1)$. Since $S_{i}$ is of signature $(1,1)$ and $\Lambda$ is of signature $(3,19), T_{i}$ are of signature $(2,18)$. By $(2.11)(1)$, we have also $l\left(A_{T_{i}}\right)=l\left(A_{S_{i}}\right) \leq 2$. Now the assertion (2) follows from $(2.11)(3)$.

Let $T_{i}(i \in\{1, \cdots, N\})$ be the sub-lattices of $\Lambda$ found in (2.12). Take an even lattice $T$ of signature $(2,18)$ which is isomorphic to all these $T_{i}$. We denote by $\varphi_{i}: T \rightarrow \Lambda$ a primitive embedding of $T$ such that $\varphi_{i}(T)=T_{i}$.

Lemma (2.13). There is a positive definite 2 -dimensional subspace $P$ of $T \otimes_{\mathbf{Z}} \mathbf{R}$ such that $P^{\perp} \cap T=\{0\}$, where $P^{\perp}$ is the orthogonal space of $P$ in $T \otimes_{\mathbf{Z}} \mathbf{R}$.

Proof. Since the signature of $T$ is $(2,18)$, there is a positive definite 2-dimensional subspace $P_{0}$ of $T \otimes_{\mathbf{z}} \mathbf{R}$. Note that positive definiteness is an open condition in 
the real Grassman manifold $\operatorname{Gr}(2, T \otimes \mathbf{z} \mathbf{R})$ in the classical topology. Moreover, in $\operatorname{Gr}\left(2, T \otimes_{\mathbf{Z}} \mathbf{R}\right)$, the locus $P^{\perp} \cap T \neq\{0\}$ is countable union of the proper Zariski closed subsets $(P, t)=0(t \in T-\{0\})$ in $\operatorname{Gr}\left(2, T \otimes_{\mathbf{Z}} \mathbf{R}\right)$. Here the properness is because $T$ is non-degenerate. Therefore, there is a desired $P$ near $P_{0}$ in $\operatorname{Gr}\left(2, T \otimes_{\mathbf{Z}} \mathbf{R}\right)$.

(2.14) Let us complete the proof of (1.7). Let $P$ be the space found in (2.13). Let $\left\langle\eta_{1}, \eta_{2}\right\rangle$ be the orthonomal basis of $P$. Set $\omega:=\eta_{1}+\sqrt{-1} \eta_{2}$ in $P \otimes_{\mathbf{R}} \mathbf{C}$. Then, by $\left(\eta_{1}, \eta_{1}\right)=\left(\eta_{2}, \eta_{2}\right)=1$ and $\left(\eta_{1}, \eta_{2}\right)=0$, one has $(\omega, \omega)=0$ and $(\omega, \bar{\omega})=2>0$. Here $\bar{\omega}$ is the complex conjugate of $\omega$ with respect to the real structure $P$. Therefore if we set $\omega_{i}=\varphi_{i}(\omega) \in \Lambda \otimes_{\mathbf{Z}} \mathbf{C}$, then $\omega_{i} \in T_{i} \otimes_{\mathbf{Z}} \mathbf{C}$, $\left(\omega_{i}, \omega_{i}\right)=0$ and $\left(\omega_{i}, \bar{\omega}_{i}\right)>0$ as well. Moreover, by $(2.13)$ and by $\Phi_{i}\left(S_{i}\right)=T_{i}^{\perp}$ in $\Lambda$, the following equalities hold in $\Lambda \otimes_{\mathbf{Z}} \mathbf{C}$ :

$$
\begin{gathered}
\omega_{i}^{\perp} \cap \Lambda=\left\langle\varphi_{i}\left(\eta_{1}\right), \varphi_{i}\left(\eta_{2}\right)\right\rangle^{\perp} \cap \Lambda \\
=\varphi_{i}(P)^{\perp} \cap \Lambda=T_{i}^{\perp} \cap \Lambda=\Phi_{i}\left(S_{i}\right) .
\end{gathered}
$$

Therefore $\left(\Lambda, \mathbf{C} \omega_{i}\right)(i \in\{1, \cdots, N\})$ define the weight two Hodge structures on $\Lambda$ such that $\omega_{i}^{\perp}=\Phi_{i}\left(S_{i}\right)$. Then, by the surjectivity of the period mapping of K3 surfaces and by the Lefschetz $(1,1)$ - Theorem, there are marked K3 surfaces $\left(X_{i}, \tau_{i}\right)$ such that $\tau\left(\mathbf{C} \omega_{X_{i}}\right)=\mathbf{C} \omega_{i}$ and $\tau_{i}\left(\mathrm{NS}\left(X_{i}\right)\right)=\Phi_{i}\left(S_{i}\right)$. This implies $\tau_{i}\left(T_{X_{i}}\right)=T_{i}$ as well. Since $\Phi_{i}\left(S_{i}\right)$ are hyperbolic, so are $N S\left(X_{i}\right)$. Therefore $X_{i}$ are projective. Moreover, by the construction, the following homomorphism gives a Hodge isometry between $\left(T_{X_{i}}, \mathbf{C} \omega_{X_{i}}\right)$ and $\left(T_{X_{j}}, \mathbf{C} \omega_{X_{i}}\right)$ :

$$
T_{X_{i}} \stackrel{\tau_{i}}{\longrightarrow} \varphi_{i}\left(T_{i}\right) \stackrel{\varphi_{i}^{-1}}{\longrightarrow} T \stackrel{\varphi_{j}}{\longrightarrow} \varphi_{j}\left(T_{j}\right) \stackrel{\tau_{j}^{-1}}{\longrightarrow} T_{X_{j}} .
$$

These K3 surfaces $X_{i}(i \in\{1, \cdots, N\})$ satisfy all the requirement in (1.7).

\section{$\S 3$. Proof of (1.9)}

Let $(T, \mathbf{C} \omega)$ be a lattice with weight two Hodge structure isomorphic to the Hodge structure $\left(T_{X}, \mathbf{C} \omega_{X}\right)$. (For this notation, we note that the Hodge structure on the lattice $T_{X}$ is determined by the inclusion $\mathbf{C} \omega_{X} \subset T_{X} \otimes_{\mathbf{Z}}$ C.) Let $Y \in \mathcal{S}_{T}(X)$. Then $\operatorname{rk} N S(Y)=22-\operatorname{rk} T$ and $|\operatorname{det} \mathrm{NS}(Y)|=\left|\operatorname{det} T_{Y}\right|=\left|\operatorname{det} T_{Y}\right|$. Therefore, the finiteness of reduction of non-degenerate integral quadratic forms with bounded determinant and bounded rank (see for instance [Cs, Page 128, Theorem 1.1]), one has

$$
\mid\left\{\mathrm{NS}(Y) \mid Y \in \mathcal{S}_{T}(X)\right\} / \text { isom } \mid<\infty .
$$

Let $S_{i}(i \in\{1, \cdots, N\})$ be the complete representative of the set above. Then one has $\mathcal{S}_{T}(X)=\cup_{i=1}^{N} \mathcal{S}_{i}(X)$ (disjoint union), where

$$
\mathcal{S}_{i}(X):=\left\{Y \in \mathcal{S}_{T}(X) \mid \mathrm{NS}(Y) \simeq S_{i}\right\} / \text { isom } .
$$


Therefore, it suffices to show that $\left|\mathcal{S}_{i}(X)\right|<\infty$ for each $i$. Choose $i$. For simplicity of notation, we write $S, \mathcal{S}(X)$ for $S_{i}, \mathcal{S}_{i}(X)$ and so on from now.

A Z-module $L$ such that $S \oplus T \subset L \subset S^{*} \oplus T^{*}$ is called an over lattice of $S \oplus T$. Let us consider all the even unimodular over lattices $L$ of $S \oplus T$ such that $S$ and $T$ are both primitive in $L$. Such an $L$ is an even unimodular lattice of signature $(3,19)$. Therefore $L$ is isomorphic to the K3 lattice. Since $\left(S^{*} \oplus T^{*}\right) /(S \oplus T)=S^{*} / S \oplus T^{*} / T$ is a finite group, there are only finitely many such $L$ as a subset of $S^{*} \oplus T^{*}$. We write all of them by $L_{j}(j \in\{1, \cdots, M\})$.

Let $Y \in \mathcal{S}(X)$. Then, we have an isometry $f: \mathrm{NS}(Y) \rightarrow S$ and a Hodge isometry $g:\left(T_{Y}, \mathbf{C} \omega_{Y}\right) \rightarrow(T, \mathbf{C} \omega)$. This induces an isomorphism $(f \oplus g): S_{Y}^{*} \oplus$ $T_{Y}^{*} \rightarrow S^{*} \oplus T^{*}$. Then there is $j \in\{1, \cdots, M\}$ such that $(f \oplus g)\left(H^{2}(Y, \mathbf{Z})\right)=L_{j}$. Write $\tau_{Y}:=(f \oplus g) \mid H^{2}(Y, \mathbf{Z})$. Conversely, by the surjectivity of the period mapping of K3 surfaces, for each $j \in\{1, \cdots, M\}$, there is a marked K3 surface $\left(X_{j}, \tau_{X_{j}}\right)$ such that $\tau_{X_{j}}: H^{2}\left(X_{j}, \mathbf{Z}\right) \rightarrow L_{j}$ is a Hodge isometry in the sense that $\tau_{j}\left(\omega_{X_{j}}\right)=\omega$. Since $T$ and $S$ are both primitive in $L_{j}$, we have $\tau_{X_{j}}\left(T_{X_{j}}\right)=T$ and $\tau_{X_{j}}\left(\mathrm{NS}\left(X_{j}\right)\right)=S$ in this case. Moreover, if we put $f=\tau_{X_{j}} \mid \operatorname{NS}\left(X_{j}\right)$ and $g=$ $\tau_{X_{j}} \mid T_{X_{j}}$, then $\tau_{X_{j}}$ is recovered from $f$ and $g$ by the process explained above. Let us choose for each $j \in\{1, \cdots, M\}$ a marked K3 surface $\left(X_{j}, \tau_{X_{j}}\right)$ as above. Let $Z \in \mathcal{S}(X)$. Then $\tau_{Z}\left(H^{2}(Z, \mathbf{C})\right)=L_{j}$ for some $j \in\{1, \cdots, M\}$. By construction, we have $\tau_{Z}\left(H^{2}(Z, \mathbf{Z})\right)=L_{j}=\tau_{X_{j}}\left(H^{2}\left(Y_{j}, \mathbf{Z}\right)\right)$ and $\tau\left(\mathbf{C} \omega_{Z}\right)=\mathbf{C} \omega=\tau_{X_{j}}\left(\mathbf{C} \omega_{X_{j}}\right)$. Thus, $\tau_{Z}^{-1} \circ \tau_{X_{j}}: H^{2}\left(X_{j}, \mathbf{Z}\right) \rightarrow H^{2}(Z, \mathbf{Z})$ is a Hodge isometry. By the global Torelli Theorem for K3 surface, we have then $Z \simeq X_{j}$. Therefore $\mathcal{S}(X)$ consists of at most $M$ elements.

\section{$\S 4$. Proof of (1.10)}

Idea of proof of (1.10) is similar to that of (1.9), but we need a bit more precise argument in order to obtain the exact number $\left|\mathcal{S}_{T}(X)\right|$.

Let $S=\langle l\rangle$ be a lattice of rank 1 such that $\left(l^{2}\right)=2 n$. Let $X$ be the same as in (1.10). As before, we choose an abstract lattice with weight two Hodge structure $(T, \mathbf{C} \omega)$ isomorphic to $\left(T_{X}, \mathbf{C} \omega_{X}\right)$. If $Y \in \mathcal{S}_{T}(X)$, then $\mathrm{NS}(Y)=\mathbf{Z} l_{Y}$ and $\left(l_{Y}\right)^{2}=\left|\operatorname{det} T_{Y}\right|=\left|\operatorname{det} T_{X}\right|=\left(l_{X}^{2}\right)=2 n$. We take $l_{Y}$ the ample class. So, $l_{Y}$ is uniquely determined by $Y$. We have an isometry $f_{Y}: \mathrm{NS}(Y) \simeq S ; l_{Y} \mapsto l$ and a Hodge isometry $g_{Y}:\left(T_{Y}, \mathbf{C} \omega_{Y}\right) \rightarrow(T, \mathbf{C} \omega)$. We choose and fix $f_{Y}$ and $g_{Y}$ for each $Y \in \mathcal{S}_{T}(X)$.

Lemma (4.1). Hodge isometry from $\left(T_{Y}, \mathbf{C} \omega_{Y}\right)$ to $(T, \mathbf{C} \omega)$ is either $g_{Y}$ or $-g_{Y}$. Conversely both are Hodge isometries.

Proof. The last statement is clear. Let us show the first assertion. Let $g$ : $\left(T_{Y}, \mathbf{C} \omega_{Y}\right) \rightarrow(T, \mathbf{C} \omega)$ be a Hodge isometry. Then $g_{Y}^{-1} \circ g$ is a Hodge isometry of $\left(T_{Y}, \mathbf{C} \omega_{Y}\right)$. So, it suffices to show that if $h$ is a Hodge isometry of $\left(T_{Y}, \mathbf{C} \omega_{Y}\right)$, then $h=i d$ or $-i d$.

Since $T \otimes_{\mathbf{Z}} \mathbf{R}=P \oplus N$, where $P=\left(\mathbf{C} \omega_{Y} \oplus \mathbf{C} \overline{\omega_{Y}}\right) \cap\left(T \otimes_{\mathbf{Z}} \mathbf{R}\right)$ and $N=$ $H^{1,1}(Y, \mathbf{C}) \cap\left(T \otimes_{\mathbf{Z}} \mathbf{R}\right)$. Here $P$ is positive definite. Since $Y$ is projective, $N$ 
is negative definite by the Hodge index Theorem. Since $h$ is a Hodge isometry, $h \in O(P) \times O(N)$. Here $O(*)$ is the orthogonal group. Since $P$ and $N$ are both definite, $h$ is diagonalizable and the eigenvalues of $h$ (in $\mathbf{C}$ ) are all absolute value 1 . On the other hand, since $h$ is defined over $\mathbf{Z}$, the eigenvalues of $h$ are all algebraic integers. Therefore, the eigenvalues of $h$ are all root of unity by the Theorem of Kronecker. In particular, there is a natural number $I$ such that $h\left(\omega_{Y}\right)=\zeta_{I} \omega_{Y}$, where $\zeta_{I}$ is a primitive $I$-th root of unity. Then $\operatorname{ord}(h)=I$. Otherwise, we have $\operatorname{ord}(h)=k I$ for some integer $k \geq 2$. However, since $h$ is defined over $\mathbf{Z}$, the space $T^{\prime}=\left\{x \in T_{Y} \mid h^{I}(x)=x\right\}$ would be a primitive submodule of $H^{2}(Y, \mathbf{Z}), T^{\prime} \neq T$ and $\omega_{Y} \in T^{\prime} \otimes_{\mathbf{Z}} \mathbf{C}$. However, this contradicts the direct consequence of the Lefschetz $(1,1)$-Theorem: $T_{Y}$ is the minimal primitive sub-module of $H^{2}(Y, \mathbf{Z})$ such that $\omega_{Y} \in T_{Y} \otimes_{\mathbf{Z}} \mathbf{C}$.

On the other hand, since $\operatorname{dim} N=\operatorname{rk} T_{Y}-2=19$ is odd, $h \mid N$ and therefore $h$ has 1 or -1 as its eigenvalue. Since $h$ is defined over $T_{Y}$ and \pm 1 is rational, there then exists an element $a \in T_{Y}-\{0\}$ such that either $h(a)=a$ or $h(a)=-a$. Therefore we have two cases:

(1) $h$ has eigenvalue 1 ;

(2) $h$ does not have eigenvalue 1 but has eigen value -1 .

We shall show that in the first case $h=i d$ and in the second case $h=-i d$.

Let us consider the Case 1. Take $a \in T_{Y}-\{0\}$ such that $h(a)=a$. Then:

$$
\left(\zeta_{I} \omega_{Y}, a\right)=\left(h\left(\omega_{Y}\right), h(a)\right)=\left(\omega_{Y}, a\right) .
$$

If $I \neq 1$, this would imply $\left(\omega_{Y}, a\right)=0$. Then $a \in \mathrm{NS}(Y) \cap T_{Y}=\{0\}$, a contradiction. Therefore $I=1$ and $h=i d$.

Let us consider the Case 2. There is $a \in T_{Y}-\{0\}$ such that $h(a)=-a$. Then the formula

$$
\left(\zeta_{I} \omega_{Y},-a\right)=\left(h\left(\omega_{Y}\right), h(a)\right)=\left(\omega_{Y}, a\right)
$$

implies $I=2$. Indeed, otherwise, we would have $\left(\omega_{Y}, a\right)=0$ and get the same contradiction as Case 1. From this and the case assumption, the eigenvalues of $h$ are all -1 . Since $h$ is diagonalizable, this implies $h=-i d$.

As in Section 3, we take all the even unimodular over lattices $L$ of $S \oplus T$ such that $S$ and $T$ are both primitive in $L$. Write all of them by $L_{j}(j \in\{1, \cdots, M\})$. As in the proof of (1.9), if $Y \in \mathcal{S}_{T}(X)$, then under the natural extension $\left(f_{Y} \oplus\right.$ $\left.g_{Y}\right): \mathrm{NS}(Y)^{*} \oplus T_{Y}^{*} \rightarrow S^{*} \oplus T^{*}$, there is $j$ such that $\left(f_{Y} \oplus g_{Y}\right)\left(H^{2}(Y, \mathbf{Z})\right)=L_{j}$. We write $\tau_{Y}:=\left(f_{Y} \oplus g_{Y}\right) \mid H^{2}(Y, \mathbf{Z})$ as before. We already observed the following fact in Section 3:

\section{Lemma (4.2).}

(1) For each $j \in\{1, \cdots, M\}$, there is a K3 surface $X_{j} \in \mathcal{S}_{T}(X)$ such that $\tau_{X_{j}}\left(H^{2}\left(X_{j}, \mathbf{Z}\right)\right)=L_{j}$. We fix such an $X_{j}$ for each $j$ in what follows.

(2) For each $Y \in \mathcal{S}_{T}(X)$, there is $j \in\{1, \cdots, M\}$ such that $Y \simeq X_{j}$. 
So, one can find complete representatives of $\mathcal{S}_{T}(X)$ in $\left\{X_{j}\right\}_{j=1}^{M}$.

However, by (4.1), there are exactly two choices of $g_{X_{j}}$ for each $X_{j} \in \mathcal{S}_{T}(X)$ : $g_{X_{j}}$ and $-g_{X_{j}}$. Therefore in order to find out the complete representatives in $\left\{X_{j}\right\}_{j=1}^{M}$, we also need to seek how $L_{j}$ changes when we replace $g_{X_{j}}$ by $-g_{X_{j}}$.

Let $L$ be any one of $L_{j}$. Then $T^{*} / T \simeq L /(T \oplus S) \simeq S^{*} / S \simeq \mathbf{Z} / 2 n$ by (2.11)(1). Therefore, $S^{*} / S=\left\langle\frac{l}{2 n}\right\rangle$ and there is $t \in T$ such that $T^{*} / T=\left\langle\frac{t}{2 n}\right\rangle$. This $t$ is independent of $L$. Note that $L /(T \oplus S)=\left\langle\frac{a l+b t}{2 n}\right\rangle$ for some $a, b \in \mathbf{Z}$. This is because $\mathbf{Z} / 2 n \simeq L /(T \oplus S) \subset S^{*} / S \oplus T^{*} / T=\left(S^{*} \oplus T^{*}\right) /(S \oplus T)$. Note also that under the natural isomorphism $L /(T \oplus S) \simeq S^{*} / S$ and $L /(T \oplus S) \simeq T^{*} / T$, $\frac{a l+b t}{2 n}$ is mapped to $\frac{a l}{2 n}$ and $\frac{b t}{2 n}$. Therefore, $(a, 2 n)=(b, 2 n)=1$. So, we may write from the first that $L /(T \oplus S)=\left\langle\frac{l+b t}{2 n}\right\rangle$, where $(b, 2 n)=1$. Note that there is a natural one to one correspondence between over lattices of $S \oplus T$ and the subgroups of $\left(S^{*} \oplus T^{*}\right) /(S \oplus T)=S^{*} / S \oplus T^{*} / T$. Therefore $b \bmod 2 n$ is uniquely determined by $L$.

Lemma (4.3). Let $Y \in \mathcal{S}_{T}(X)$.

(1) Assume that $n \neq 1$. Then $\left(f_{Y} \oplus g_{Y}\right)\left(H^{2}(Y, \mathbf{Z})\right) \neq\left(f_{Y} \oplus\left(-g_{Y}\right)\right)\left(H^{2}(Y, \mathbf{Z})\right)$ and there exists unique $i=i(Y) \in\{1, \cdots, M\}$ such that $\tau_{X_{i}}\left(H^{2}\left(X_{i}, \mathbf{Z}\right)\right)=$ $\left(f_{Y} \oplus\left(-g_{Y}\right)\right)\left(H^{2}(Y, \mathbf{Z})\right)$.

(2) Assume that $n=1$. Then $\left(f_{Y} \oplus g_{Y}\right)\left(H^{2}(Y, \mathbf{Z})\right)=\left(f_{Y} \oplus\left(-g_{Y}\right)\right)\left(H^{2}(Y, \mathbf{Z})\right)$.

Proof. We can write $H^{2}(Y, \mathbf{Z}) /\left(\mathrm{NS}(Y) \oplus T_{Y}\right)=\left\langle\frac{l_{Y}+b t_{Y}}{2 n}\right\rangle$, where $l_{Y}$ is the same as before and $t_{Y}$ is an element of $T_{Y}$ such that $g_{X}\left(t_{Y}\right)=t$ and $b$ is an integer such that $(b, 2 n)=1$. Then, $\left(f_{Y} \oplus g_{Y}\right)\left(H^{2}(Y, \mathbf{Z})\right) /(S \oplus T)=\left\langle\frac{l+b t}{2 n}\right\rangle$ and $\left(f_{Y} \oplus-g_{Y}\right)\left(H^{2}(Y, \mathbf{Z})\right) /(S \oplus T)=\left\langle\frac{l-b t}{2 n}\right\rangle$. Then, $\left(f_{Y} \oplus g_{Y}\right)\left(H^{2}(Y, \mathbf{Z})\right)=$ $\left(f_{Y} \oplus-g_{Y}\right)\left(H^{2}(Y, \mathbf{Z})\right)$ in $S^{*} \oplus T^{*}$ if and only if $2 b \equiv 0 \bmod 2 n$. Since $(b, 2 n)=1$, this implies $2 \equiv 0 \bmod 2 n$. However, this is possible only when $n=1$. Thus, we get the first part of (1). The last part of (1) is nothing but the definition of the set $\left\{X_{j}\right\}_{j=1}^{M}$. If $n=1$, one has $b=-b \bmod 2$ and the two lattices are the same in $S^{*} \oplus T^{*}$.

\section{Lemma (4.4).}

(1) For each $j \in\{1, \cdots, M\}$, there is exactly one $i \in\{1, \cdots, M\}$ such that $i \neq j$ and such that $X_{i} \simeq X_{j}$ when $n \neq 1$. When $n=1, X_{i} \simeq X_{j}$ if and only if $i=j$.

(2) In particular, $\left|\mathcal{S}_{T}(X)\right|=M / 2$ if $n \neq 1$ and $\left|\mathcal{S}_{T}(X)\right|=M$ if $n=1$.

Proof. (2) follows from (1) and (4.2). Let us show (1). If $n \neq 1$, then setting $\rho_{j}:=\left(f_{X_{j}} \oplus-g_{X_{j}}\right) \mid H^{2}\left(X_{j}, \mathbf{Z}\right)$, one has $\rho_{j}\left(H^{2}\left(X_{j}, \mathbf{Z}\right)\right)=L_{i(j)}$ for some $i(j) \neq j$ by (4.3). For this $i=i(j)$, the map $\tau_{i}^{-1} \circ \rho_{j}: H^{2}\left(X_{j}, \mathbf{Z}\right) \rightarrow H^{2}\left(X_{i}, \mathbf{Z}\right)$ is a Hodge isometry. Therefore $X_{i} \simeq X_{j}$ by the global Torelli Theorem.

Assume that $X_{k} \simeq X_{j}$. Let $\varphi: X_{k} \rightarrow X_{j}$ be an isomorphism. Note that $l_{X_{k}}$ is the unique ample generator of $\operatorname{NS}\left(X_{k}\right)$. Then one has $\varphi^{*}\left(l_{X_{j}}\right)=l_{X_{k}}$, 
$\varphi^{*}\left(T_{X_{j}}\right)=T_{X_{k}}$ and $\varphi^{*}\left(H^{2}\left(X_{j}, \mathbf{Z}\right)\right)=H^{2}\left(X_{k}, \mathbf{Z}\right)$. Set $\rho:=\tau_{X_{k}} \circ \varphi^{*}$ This $\rho$ is a Hodge isometry and satisfies

$$
\rho\left(l_{X_{j}}\right)=\tau_{X_{k}}\left(l_{k}\right)=l \text { and } \rho\left(H^{2}\left(X_{j}, \mathbf{Z}\right)\right)=\tau_{X_{k}}\left(H^{2}\left(X_{k}, \mathbf{Z}\right)\right)=L_{k} .
$$

On the other hand, by (4.1), we have exactly two Hodge isometries from $H^{2}\left(X_{j}, \mathbf{Z}\right)$ into $S^{*} \oplus T^{*}$ which mapps $l_{X_{j}}$ to $l$. They are $\left(f_{X_{j}}, g_{X_{j}}\right)$ and $\left(f_{X_{j}},-g_{X_{j}}\right)$. Thus, $\rho=\left(f_{X_{j}}, g_{X_{j}}\right)$ or $\rho=\left(f_{X_{j}},-g_{X_{j}}\right)$. In the first case, we have $L_{k}=L_{j}$ and in the second case we have $L_{k}=L_{i(j)}$. By the definition of $L_{*}$, we have then $k=j$ and $k=i(j)$ respectively. Then, by the definition of the set $\left\{X_{i}\right\}_{i=1}^{M}$, we have $X_{k}=X_{j}$ and $X_{k}=X_{i(j)}$ respectively. This completes the proof of (1) in the case $n \neq 1$. Proof for the case $n=1$ is exactly the same.

In order to complete the proof of (1.10), it now suffices to prove the following Lemma:

Lemma (4.5). $M=\left(\left|\left((\mathbf{Z} / 4 n)^{\times}\right)_{2}\right|\right) / 2$.

Proof. Recall that $M$ is the cardinality of the set consisting of the even unimodular over lattices $L$ of $S \oplus T$ such that $S$ and $T$ are both primitive in $L$. We write this set by $\mathcal{M}$.

Let $L \in \mathcal{M}$. Then, as remarked before (4.3), taking an element $t$ such that $T^{*} / T=\left\langle\frac{t}{2 n}\right\rangle$, we can write $L /(T \oplus S)=\left\langle\frac{l+b t}{2 n}\right\rangle$. Then $\bar{b}:=b \bmod 2 n$ is an element of $(\mathbf{Z} / 2 n)^{\times}$and is uniquely determined by $L$.

Conversely, if an over lattice $L$ of $S \oplus T$ satisfies $L /(S \oplus T)=\left\langle\frac{l+b t}{2 n}\right\rangle$ for some natural number $b$ such that $(b, 2 n)=1$, i.e. $\bar{b} \in(\mathbf{Z} / 2 n)^{\times}$, then $|\operatorname{det} L|=1$ and both $S$ and $T$ are primitive in $L$. Therefore, there is one-to-one correspondence between $\mathcal{M}$ and the set of element $\bar{b}$ of $(\mathbf{Z} / 2 n)^{\times}$such that the overlattice $S+$ $T+\left\langle\frac{l+b t}{2 n}\right\rangle$ is even and integral. We write the latter set by $\mathcal{D}$. Then $|\mathcal{M}|=|\mathcal{D}|$ and we may calculate $|\mathcal{D}|$. Since $\left(\frac{t}{2 n}, u\right) \in \mathbf{Z}$ and $\left(\frac{l}{2 n}, u\right) \in \mathbf{Z}$ if $u \in S \oplus T$ (by the definition of the dual) and since $S, T$ are both even, we see that $L=S+T+\left\langle\frac{l+b t}{2 n}\right\rangle$ is even and integral if and only if $\left(\frac{l+b t}{2 n}\right)^{2}$ is an even number. Since $\left(\frac{t}{2 n}, t\right) \in \mathbf{Z}$, we have $\left(t^{2}\right)=2 n c$ for some integer $c$. Then $b$ satisfies that

$$
\left(\frac{l+b t}{2 n}\right)^{2}=\frac{2 n+b^{2}\left(t^{2}\right)}{4 n^{2}}=\frac{1+b^{2} c}{2 n} .
$$

Thus, the condition $b \bmod 2 n \in \mathcal{D}$ is equivalent to

$$
1+b^{2} c=0 \bmod 4 n \text {, i.e. } b^{2} c=-1 \text { in } \mathbf{Z} / 4 n \text {. }
$$

There is at least one $b \bmod 2 n \in \mathcal{D}$ corresponding to the original $X$. We write this $b$ by $b_{0}$. Then $b_{0}^{2} c=-1$ in $\mathbf{Z} / 4 n$. In particular, both $b_{0}$ and $c$ are unit elements in $\mathbf{Z} / 4 n$. Therefore the condition $b \bmod 2 n \in \mathcal{D}$ is equivalent to the condition $\left(\frac{b}{b_{0}}\right)^{2}=1$ in $\mathbf{Z} / 4 n$, that is, $b$ is written as $b_{0} u$ for an integer $u$ which 
gives an element of $(\mathbf{Z} / 4 n)^{\times}$of order at most 2 . Since $b_{0}$ is invertible in $\mathbf{Z} / 4 n$, we see that $b_{0}$ is invertible in $\mathbf{Z} / 2 n$ as well. Then $b_{0} u=b_{0} v$ in $\mathbf{Z} / 2 n$ if and only if $u=v$ in $\mathbf{Z} / 2 n$, i.e. $v=u$ or $v=u+2 n$ in $\mathbf{Z} / 4 n$. Note that $u+2 n \neq u$ in $\mathbf{Z} / 4 n$. Note also that if $u$ is a unit element in $\mathbf{Z} / 4 n$, then $u+2 n$ is also a unit element in $\mathbf{Z} / 4 n$. Indeed, if $u c=1$ in $\mathbf{Z} / 4 n$, then $u=c=1$ in $\mathbf{Z} / 2$. Therefore

$$
(u+2 n)(c+2 n)=u c+2 n(u+c)+4 n^{2}=1 \text { in } \mathbf{Z} / 4 n .
$$

Hence $M=|\mathcal{D}|=\left|\left\{b_{0} u \bmod 2 n\right\}\right|=\left|\left((\mathbf{Z} / 4 n)^{\times}\right)_{2}\right| / 2$.

Now combining (4.4) and (4.3), we obtain (1.10). The explicit formula follows from the Chinese remainder Theorem and the fact $\left(\mathbf{Z} / 2^{2}\right)^{\times} \simeq \mathbf{Z} / 2,\left(\mathbf{Z} / 2^{a}\right)^{\times} \simeq$ $\mathbf{Z} / 2 \oplus \mathbf{Z} / 2^{a-2}$ if $a \geq 3$ and $\left(\mathbf{Z} / p^{e}\right)^{\times} \simeq \mathbf{Z} / p^{e-1}(p-1)$ if $p \geq 3$ is a prime.

\section{Appendix}

In this appendix, we shall remark a generalization of (1.7) for hyperkähler manifold of higher dimension.

By a hyperkähler manifold, we mean a simply-connected compact Kähler manifold $X$ which admits a everywhere non-degenerate holomorphic 2 -form $\omega_{X}$ and satisfies $H^{2,0}(X)=\mathbf{C} \omega_{X}$. Let $X$ be a hyperkähler manifold. $X$ is of even dimensional. By Beauville [Be], $X$ admits a non-degenerate primitive integral symmetric bilinear form $(*, *): H^{2}(X, \mathbf{Z}) \times H^{2}(X, \mathbf{Z}) \rightarrow \mathbf{Z}$ of signature $\left(3, B_{2}(X)-3\right)$. By his construction, this bilinear form together with the Hodge decomposition $H^{2}(X, \mathbf{C})=H^{1,1}(X) \oplus \mathbf{C} \omega_{X} \oplus \mathbf{C} \omega_{X}$ forms a weight two Hodge structure on $H^{2}(X, \mathbf{Z})$. We define the transcendental lattice $T_{X}$ of $X$ to be the orthogonal lattice of $\operatorname{NS}(X) \simeq \operatorname{Pic} X$ in $H^{2}(X, \mathbf{Z})$ with respect to this bilinear form. $T_{X}$ is the minimal primitive sub-lattice of $H^{2}(X, \mathbf{Z})$ whose $\mathbf{C}$-linear extension contains $\omega_{X}$. As is remarked in [Hu1] (all of which results are now avaiable by [Hu2]), $H^{2}(X, \mathbf{Z})$ and $H^{2}\left(X^{\prime}, \mathbf{Z}\right)$ are Hodge isometric if $X$ and $X^{\prime}$ are birational.

If $X$ is the Hilbert scheme $\operatorname{Hilb}^{n}(S)$ of 0-dimensional subschemes of length $n(\geq 2)$ on a K3 surface $S$ or its (Kähler) deformation, then $X$ is a hyperkähler manifold of dimension $2 n$ and satisfies $\left(H^{2}(X, \mathbf{Z}),(*, *)\right) \simeq \Lambda \oplus\langle-2(n-1)\rangle$. Here $\Lambda$ is a K3 lattice.

Proposition A. Let $n$ be any natural number. For any given natural number $N$, there are $N$ mutually non-birational projective hyperkähler manifolds $\left\{X_{j}\right\}_{j=1}^{N}$ of dimension $2 n$ such that $\left(T_{X_{j}}, \mathbf{C} \omega_{X_{j}}\right)$ are mutually Hodge isometric but $N S\left(X_{j}\right)$ are not isomorphic to one another.

Proof. Let $S_{i}$ and $T_{i}(i=1, \cdots, N)$ be the lattices found in (2.9) and (2.12). Consider the sublattices $\tilde{T}_{i}:=T_{i} \oplus\langle-2(n-1)\rangle \subset \Lambda \oplus\langle-2(n-1)\rangle$. Then, $\tilde{T}_{i}$ are primitive, of signature $(2,19)$ and are isomorphic to one another by the construction and (2.11). (We remark that $l\left(A_{\tilde{T}_{i}}\right) \leq l\left(A_{T_{i}}\right)+1 \leq 3$ so that we can apply $(2.11)$ to see these $\tilde{T}_{i}$ are isomorphic.) Fix a lattice $\tilde{T}$ isomorphic 
to all $\tilde{T}_{i}$ and fix primitive embeddings $\tilde{\varphi}_{i}: \tilde{T} \simeq \tilde{T}_{i} \subset \Lambda \oplus\langle-2(n-1)\rangle$. As in (2. 13), we also take a positive definite 2 dimensional subspace $\tilde{P}$ of $\tilde{T} \otimes_{\mathbf{Z}} \mathbf{R}$ such that $\tilde{P}^{\perp} \cap \tilde{T}=\{0\}$. One can then obtain a weight 2 Hodge structure on $\tilde{T}$, say, $(\tilde{T}, \mathbf{C} \tilde{\omega})$ as in (2.14). Then, by applying the surjectivity of the period mapping by Huybrechts [Hu1] for the weight two Hodge structures $(\Lambda \oplus\langle-2(n-$ $\left.1)\rangle, \mathbf{C} \tilde{\varphi}_{i}(\tilde{\omega})\right)$ and repeating exactly the same argument as in (2.14), one can find for each $i$ a marked hyperkḧler manifold $\left(X_{i}, \tau_{i}\right)$ (equivalent to $\operatorname{Hilb}^{n}(S)$ under deformation) such that $\left(T_{X_{i}}, \mathbf{C} \omega_{X_{i}}\right) \simeq\left(\tilde{T}_{i}, \mathbf{C} \tilde{\varphi}_{i}(\tilde{\omega})\right)$ and $\operatorname{NS}\left(X_{i}\right) \simeq S_{i}$ via $\tau_{i}$. Since $S_{i}$ is of signature $(1,1), X_{i}$ is projective by the projectivity criterion due to Huybrechts [Hu1] (See [Hu2] for a correction of proof). Therefore, these $X_{i}$ give the result.

\section{Remark.}

(1) In [Yo], Yoshioka finds K3 surfaces $X \neq Y$ such that $\operatorname{Hilb}^{2}(X) \simeq$ $\mathrm{Hilb}^{2}(Y)$. In addition, there are several lattices $S, S^{\prime}$ and $M$ such that $S \not S^{\prime}$ but $S \oplus M \simeq S^{\prime} \oplus M$. So, Hilbert schemes of K3 surfaces found in (1.7) may not satisfy the condition of Propsition $A$.

(2) Namikawa [Nm] finds a counter example of the birational injectivity of the period mapping for hyperkähler manifolds of dimension 4. Therefore, the argument for the finiteness (1.9) in Section 3 cannot be exploited in higher dimensional hyperkähler manifolds.

\section{Acknowledgement}

This note has been grown up from a discussion with Professors S. T. Yau, S. Hosono, B. Andreas, C. H. Liu at seminar of Professor S. T. Yau. Proof owes a debt to a great help of Professor B. H. Gross, the Chair of the Department of Mathematics of Harvard University. The author would like to express his sincere thanks to all of them and to Professors R. P. Thomas and E. Kowalski for valuable discussion and comment. The author would like to express his hearty thanks to Professors J. Harris and Y. Kawamata for warm encouragement. This work has been done during his stay at Harvard University under financial support by Harvard University and Japan Monbu-Kagaku Shou (Kaigai-KenkyuKaihatsu-Doukou-Chousa) October 2001 - February 2002.

\section{References}

[Be] A. Beauville, Variétés Kähleriannes dont la premiére classe de Chern est nulle, J. Differential Geom. 18 (1983), no. 4, 755-782 (1984).

[BO] A. Bondal, D. Orlov, Reconstruction of a variety from the derived category and groups of autoequivalences, math.AG/9712029.

[BM1] T. Bridgeland, A. Maciocia, Complex surfaces with equivalent derived categories, Math. Z. 236 (2001), no. 4, 677-697.

[BM2] T. Bridgeland, A. Maciocia, Fourier-Mukai transforms for K3 and elliptic fibrations, math.AG/9908022.

[BPV] W. Barth, C. Peters, A. Van de Ven, Compact complex surfaces, Ergebnisse der Mathematik und ihrer Grenzgebiete (3) 4. Springer-Verlag, Berlin, 1984. 
[Cl] A. Caldararu, Non-fine moduli spaces of sheaves on K3 surfaces, math.AG/0108180.

[Cs] J. W. S. Cassels, Rational quadratic forms, London Mathematical Society Monographs, 13. Academic Press, Inc., London-New York, 1978.

[GM] S.I. Gelfand, Y.I. Manin, Methods of homological algebra, Springer-Verlag, Berlin, 1996.

[Hu1] D. Huybrechts, Compact Hyperkähler manifolds: Basic results, Invent. Math. 135 (1999), no. 1, 63-113.

[Hu2] D. Huybrechts, Erratum to the paper:Compact Hyperkähler manifolds: Basic results, math.AG/0106014.

[Iw] H. Iwaniec, Almost-primes represented by quadratic polynomials, Invent. Math. 47 (1978), no. 2, 171-188.

[Mu] S. Mukai, On the moduli space of bundles on K3 surfaces I, On the moduli space of bundles on $K 3$ surfaces. I. Vector bundles on algebraic varieties (Bombay, 1984), 341-413, Tata Inst. Fund. Res. Stud. Math., 11, Tata Inst. Fund. Res., Bombay, 1987.

[Nm] Yo. Namikawa, Counter-example to global Torelli problem for irreducible symplectic manifolds, math.AG/0110114.

$[\mathrm{Nr}]$ W. Narkiewicz, Elementary and analytic theory of algebraic numbers, 2nd edition, Springer-Verlag, Berlin; PWN-Polish Scientific Publishers, Warsaw, 1990.

[Ni] V. V. Nikulin, Integer symmetric bilinear forms and some of their geometric applications, Izv. Akad. Nauk SSSR Ser. Mat. 43 (1979), no. 1, 111-177, 238.

[Or] D. Orlov, Equivalences of derived categories and K3 surfaces, math.AG/9606006.

[PS] I. Piatetski-Shapiro and I. R. Shafarevich, A Torelli Theorem for algebraic surfaces of type K3, Math. USSR-Izv. 5 (1971), 547-587.

[Th] R. Thomas, A holomorphic Casson invriant for Calabi-Yau 3-folds and bundles on K3 fibrations, math.AG/9806111.

[Yo] K. Yoshioka, Moduli spaces of stable sheaves on abelian surfaces, math.AG/0009001.

Harvard University, Department of Mathematics, One Oxford Street, Cambridge, MA 02138, U.S.A.

E-mail address: keiji@math.harvard.edu 